\title{
InfoStick: an interaction device for Inter-Appliance Computing
}

\author{
Naohiko Kohtake ${ }^{1}$, Jun Rekimoto ${ }^{2}$, and Yuichiro Anzai ${ }^{1}$ \\ 1 Department of Computer Science, Keio University, \\ 3-14-1 Hiyoshi, Kohoku-ku, Yokohama 223-0061 Japan \\ kohtake@naohiko.com, anzai@aa.cs.keio.ac.jp \\ http://www naohiko.com/ \\ 2 Interaction Laboratory, Sony Computer Science Laboratories, \\ 3-14-3 Higashigotanda, Shinagawa-ku, Tokyo 141-0022 Japan \\ rekimoto@csl.sony.co.jp \\ http://www.csl.sony.co.jp/person/rekimoto.html/
}

\begin{abstract}
Many electric appliances have recently become network reachable, and we would receive better services from them if we could use them in combination. We have therefore developed a new hand-held interaction device called "InfoStick" that serves as an "information carrier" for these appliances. For example, a user can "pick up" TV program information from a web browser and "drop" it into a VCR deck, just like moving a physical object from one place to another. Using attached visual markers, the InfoStick identifies information appliances or other physical objects and gives an appropriate choice of action to the user. This paper explains the design and implementation of the InfoStick as well as several potential applications using this device.
\end{abstract}

\section{Introduction}

The network infrastructure has spread all over the world, and nowadays there are a variety of devices that can access the Internet. Internet access is no longer limited to personal computers or powerful workstations. Thanks to recent advances in digital and network technologies, many consumer electric devices such as VCRs, electric-organs or air-conditioners, as well as office appliances such as printers and LCD projectors are becoming "network reachable". We call these devices "Information Appliances". It is now reasonable to expect these appliances to communicate with each other in order to provide better services to users. For example, a VCR could receive a TV program information from the web browser or a printer could create a hardcopy of an image projected on a LCD projector.

However, operating these multiple devices may cause user interface problems. We might have to handle a number of remote controllers for each device, and there are few practical ways of controlling two or more appliances. When we want to "transfer" TV program information from the web browser on a computer to 
the VCR deck, the VCR controller does not help us. We thus need to operate information appliances in combination, that is not on their own appliance.

In addition, we also need to deal with physical (non-electric) objects such as printed paper. Even if digital and network technology becomes more advantageous, paper still has significant advantages: it is portable, writable, inexpensive, and physically visible. However, transferring data between information appliances and physical objects always requires manual operations. For instance, when we find an URL of an interesting web site on a poster, it is necessary to input the URL into a computer with a keyboard or write it down if there is no computer nearby. It would be quite useful if we could "pick up" a printed URL and "drop" it on an information appliance. In summary, we always need support for exchanging information between digital and physical objects. We call such operations "Inter-Appliance Computing".

To provide this support, we have developed a hand-held device called "InfoStick" that serves as "an information carrier" for Inter-Appliance Computing. Using the InfoStick, a user can "pick up" TV program information from a web browser and "drop" it into a VCR deck. The InfoStick identifies information appliances or other physical objects by recognizing attached visual markers and gives an appropriate choice of actions to the user.

\section{InfoStick Device}

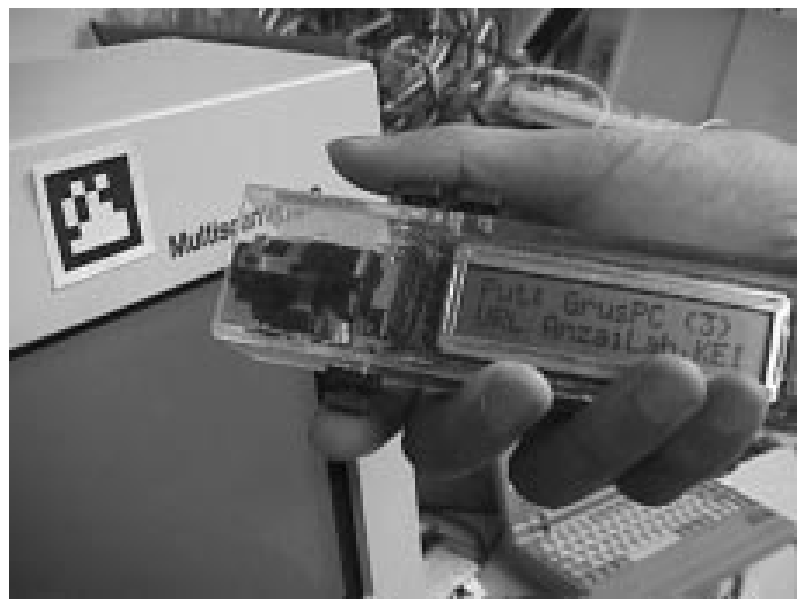

Fig. 1. External appearance of the InfoStick

The InfoStick prototype is a hand-held interaction device for exchanging information among information appliances and physical objects. Figure 1 shows 
the external appearance of the InfoStick. It consists of a small display to show what kind of data items can be exchanged, a video camera for object recognition, three buttons to operate data exchanges, and a micro processor for controlling all of them. The InfoStick can be connected to the Internet through a wireless network. Physically, it looks like a laser-pointer or a small wand, and can be easily pointed to target objects. Using the InfoStick is similar to drag-and-drop, a commonly used technique for interacting with GUIs.

The InfoStick automatically identifies the information appliance (e.g., a VCR) or the physical object (e.g., paper) in front of it, and shows a user a list of appropriate actions on the display. Visual markers attached to the objects are recognized by the InfoStick's camera. Among the three buttons for operating the InfoStick, the "get" button is used to "pick up" information from the target object, the "put" button is used to transfer information from the InfoStick to the target object, and the "select" button is used to select actions and information showed on the InfoStick display.

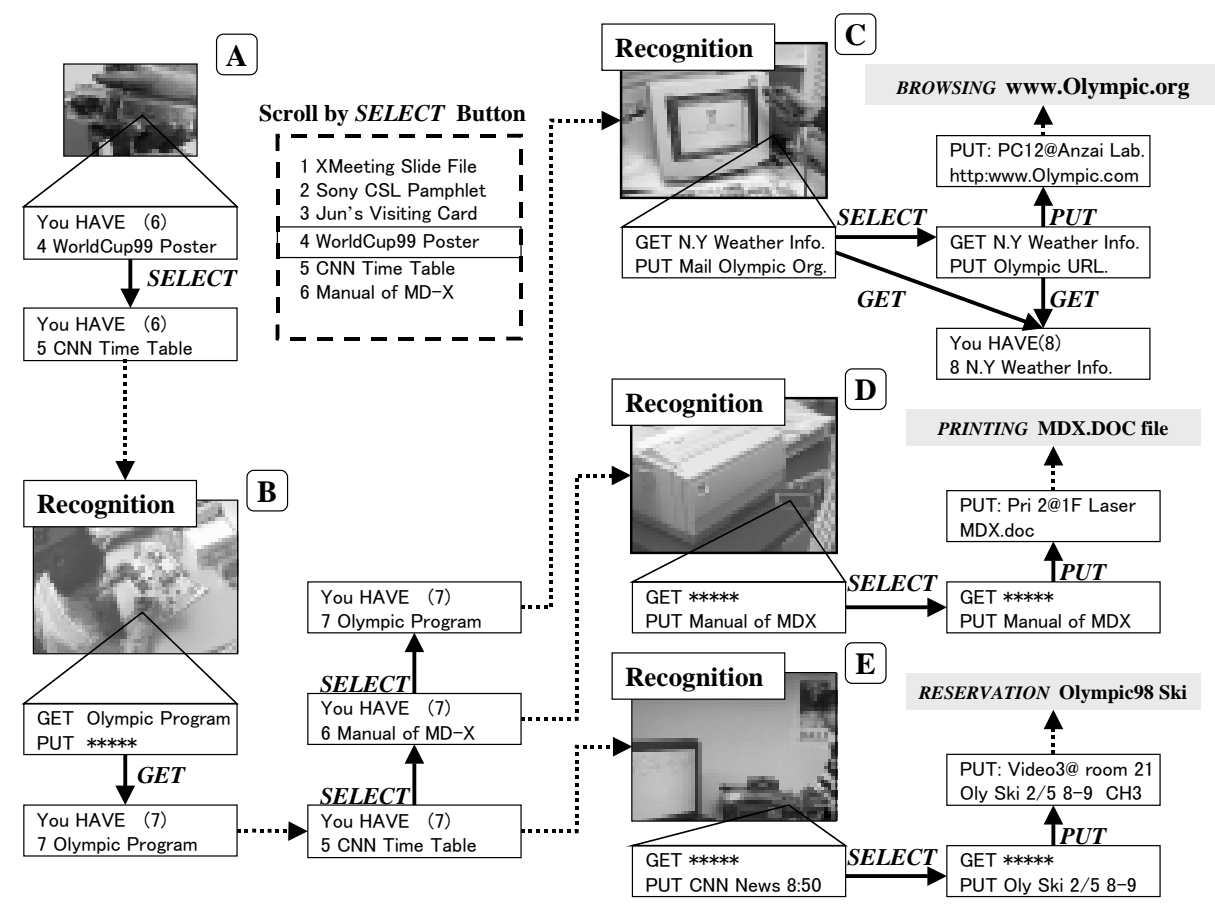

Fig. 2. Typical Information flow during the InfoStick operation 
Figure 2 shows a typical information flow during the InfoStick operation. The InfoStick display shows a list of data items, and a user can scroll down the list and select one by pushing the "select" button (Figure $2 \mathrm{~A}$ ). When a user points the InfoStick at a target object, the video camera mounted in the InfoStick detects a visual marker attached to the object (Figure 2 B). Then, the display on the InfoStick shows a list of items that can be picked up from the target object and also a list of items that can be transmitted to the target object. When the "get" button is pushed, the target object's information is stored in the InfoStick. In Figure. 2 B, a user is getting "Olympic Program information".

When a user moves to another target, the InfoStick recognizes it and the available actions corresponding to the recognized object appear on the display (Figure $2 \mathrm{C}, \mathrm{D}$, and E). If the InfoStick has some data that can be "put" into the computer, the user can select an action by the "select" button (from the mail address to the URL in Figure 2 C). By pushing the "put" button, the user can see on the display which target and what kind of information the InfoStick has put into the computer. In this case, the target is a PC "PC12@Anzai Lab." and the "put" information is "Olympic URL", which the user can then browse. However, if the InfoStick has only one possible action corresponding to the recognized object, the displayed action does not change even if the user pushes the "select" button (Figure $2 \mathrm{D}$ ). So in this case, the user can do only one operation; that is, printing out "Manual of MDX file". If a user wants to select information previously put in, he can select it before detecting a target object. Then, this information appears on the display as the possible action that he can "put" first (Figure $2 \mathrm{E}$ ). After that, other possible actions can be selected in turn.

During these operation sequences, the InfoStick does not directly "get" / "put" data from/to the target objects. Instead, actual data transfer occurs through the network to which all devices are connected. The InfoStick recognizes a target object according to the attached ID and issues appropriate data transfer commands to the network. In our system, we use printed 2D matrix codes (see Figure 1) as IDs. It is also possible to attach such IDs to non-electric objects.

\section{InfoStick Applications}

Using the prototype InfoStick, we have built several potential applications to accomplish an interaction for Inter-Appliance Computing. We believe this technology will become a part of functions for mobile phone and each user has his own InfoStick device in the future. Some examples of these applications are given in the following sections.

\subsection{Transferring Information between Computers}

A basic usage of the InfoStick is to transfer digital data between several computers. At a meeting, for example, the presenter often uses a projector to support his presentation (Figure 3). 

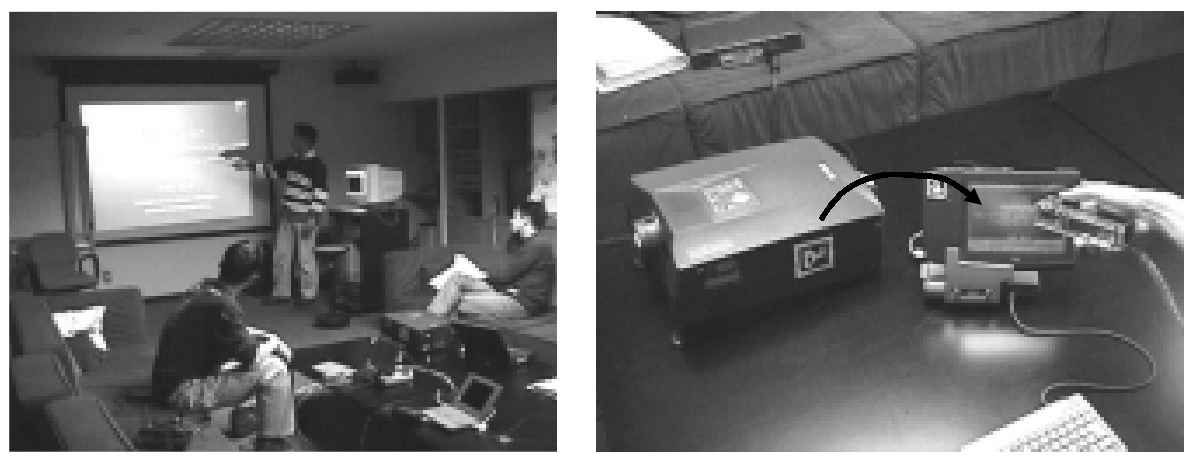

Fig. 3. At a meeting, the InfoStick creates the illusion that the presenter can "get a slide from the projector" and "put it in my computer".

The presenter can get the target presentation slide by physically pointing the InfoStick to the projector and pushing the "get" button. When he wants to take the entire slides, he can "select" the "Entire Slides" menu item and push the "get" button. After that, if he wants to create a copy of the acquired slide, he can point the InfoStick to his computer and upload the slide by pushing the "put" button. In this case, the projector itself does not have to hold the slide data. In fact, the actual slide contents are stored in servers on the network, and the projector is used as a physical landmark for obtaining data, because it has a mental connection to the currently displayed slide. The visibility and tangibility of the user's action are important because of intuitive. A user can therefore exchange information more directly and the InfoStick creates the illusion that a user can "get a slide from the projector" and "put it in my computer". On the other hand, if the usual file transfer method is used, a user must recognize both the target computer's name and the slide's name in order to move the slide by file transfer protocol (FTP). These operations are quite symbolic and thus invisible.

\subsection{Operating Information Appliances}

The second possible application of the InfoStick allows a user to operate information appliances. For example, if TV program information is stored in the InfoStick, when a user points the InfoStick to the target VCR deck, the display of the InfoStick shows the TV program names that he can reserve for recording. After selecting the program name with the "select" button and putting it into the VCR with the "put" button, the VCR is thus programmed. (Figure 4).

Another InfoStick application is making a phone call. You normally know the person's name and phone number before calling. If you do not, you have to find them out. Without the phone number, it is impossible to call. However, with the InfoStick, a user does not have to know the number because it is stored 


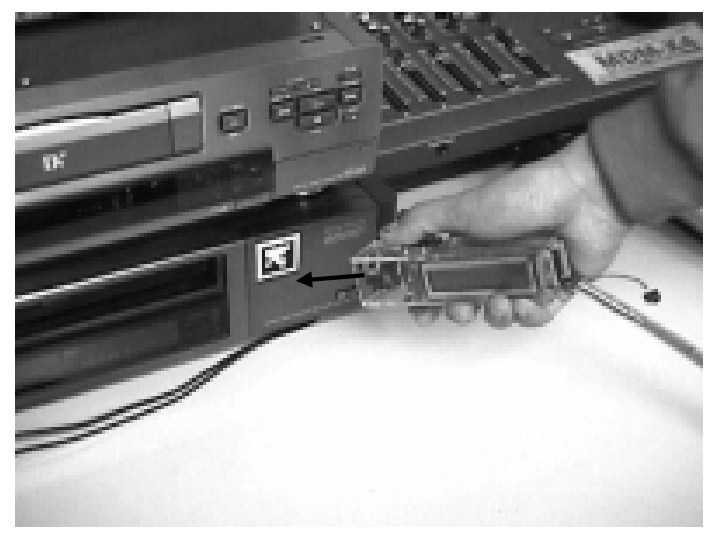

Fig. 4. The InfoStick programs a VCR to make a recording

in a server on the network. The user simply points the InfoStick at a phone and puts the person's name into the phone, which then calls the person.

\subsection{Getting information from paper}

The InfoStick can also "get" information from physical objects like paper by using visual markers. When the InfoStick recognizes the IDs, a server connected to the InfoStick displays a list of items of the recognized physical object. Then, the user can "get" the information from the target object directly by the same interaction as that with information appliances (Figure 5).
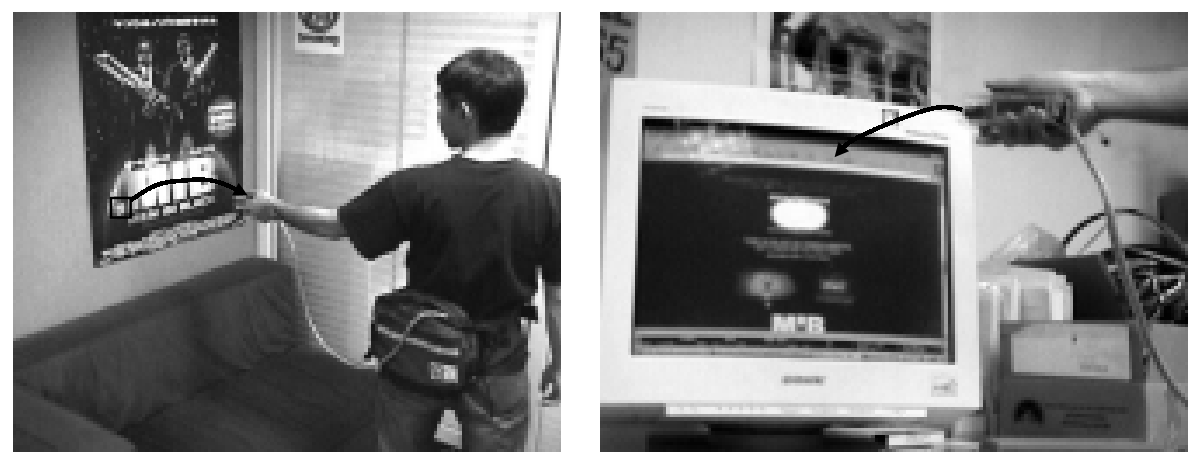

Fig. 5. A user can "pick up" a printed URL from a poster and "drop" it into a computer 


\subsection{Putting Information onto paper}

Another InfoStick application is to attach digital information onto paper like a tag or a document note. For example, it would be useful if we could attach presentation slide files to the corresponding document. Using the InfoStick, the user can "pick up" the slide data from the computer and attach it to the printed marker on the document. For instance, a user can simply take this document to the conference, "get" the presentation file from this marker, and "put" the file into the projector. So the user does not have to bring a computer, a projector or a floppy disk. InfoStick can be used to place all the necessary information on the presentation paper. The InfoStick can also use a piece of paper as a physical memory bank. If we want to store data for a long time, we can "put" it and write down its name as a title on a tag. Later, when a user wants to use this data, he can "get" it from the tag. This system can also be used by teachers for announcing information about exercises to students. After teachers "put" data with the title of the exercise on a tag, students use the InfoStick to "get" it from the tag on a notice board.

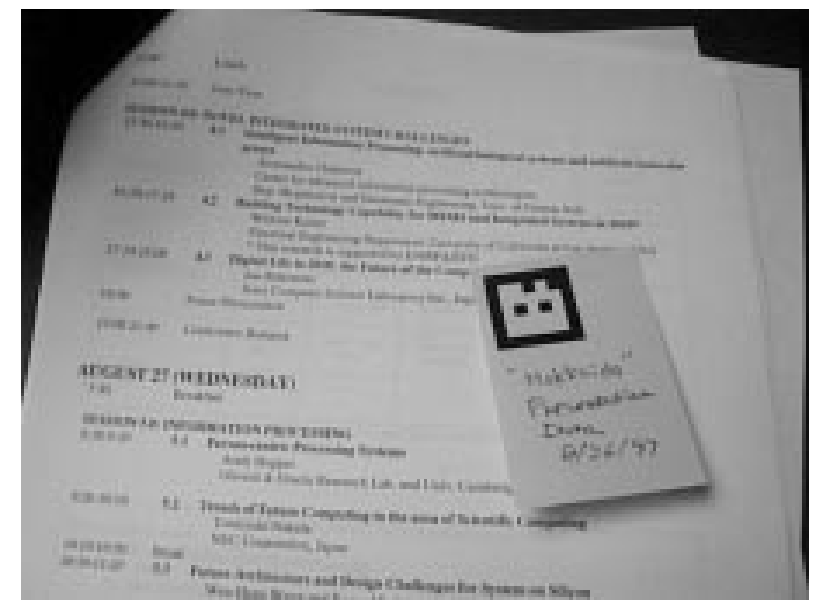

Fig. 6. A PostIt note with attached digital data

It is also possible to use a PostIt note with a printed visual marker (Figure 6). The user can attach any digital data on a PostIt note, and stick it to any objects. This usage provides a way of organizing digital data with physical documents. So even networking becomes ubiquitous, paper will still remain significant. This application of the InfoStick therefore augments electric features with advantages of paper. 


\subsection{Getting information from one object and Putting it into many objects}

In everyday life, there are many kinds of information. A name card is a typical example of this. Generally, it gives a person's name, occupation, address, phone/facsimile number, email and URL. When we make a phone call, we intuitively select appropriate information (a phone number) from the card. When we use a facsimile, the other attribute (a facsimile number) would be used instead of a phone number. There is thus an implicit correspondence between the target device and these attributes. When the InfoStick identifies the target object, it automatically selects appropriate information by combining the attribute's ID and the target object's ID (Figure 7).

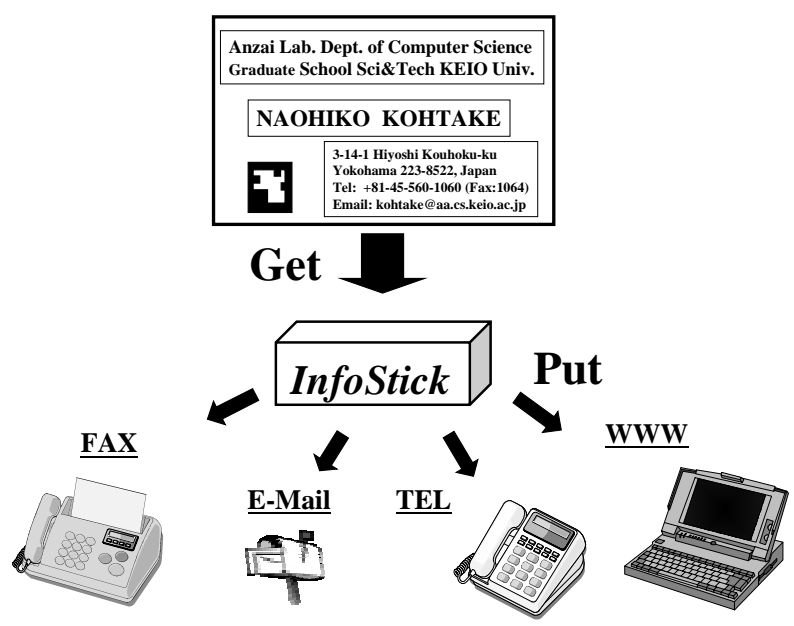

Fig. 7. Automatic selections of information according to the target object

\subsection{Getting information from a screen}

The InfoStick can recognize visual markers displayed on a screen. It is therefore possible to "pick up" the IDs from TV programs or web pages. Figure 8 illustrates this technique. When a user is watching a TV program, he finds an interesting piece of information on the TV screen. Then, he "gets" this information from the visual marker on the TV screen (above-left) and "puts" it to the nearby computer (below-left). The corresponding web page appears on the computer screen. After browsing the information from this page, he also find some information on an interesting TV program and he "gets" it by pointing the InfoStick at the browser on the screen (below-right). Finally, he goes to the VCR deck, and "puts" it into the VCR to record the TV program (above-right). 


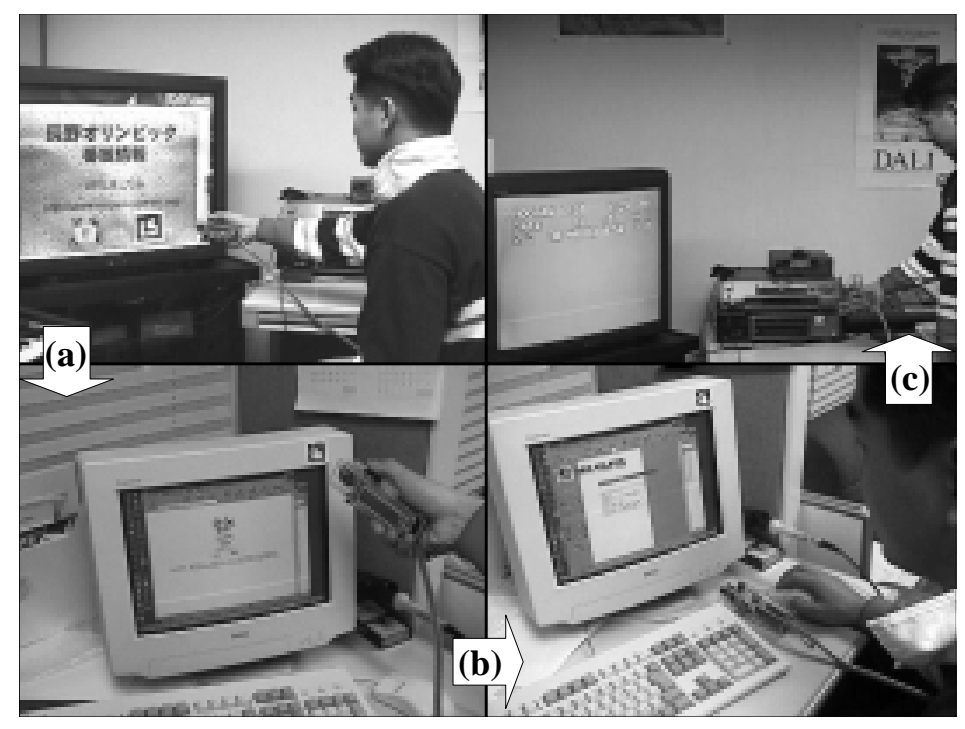

Fig. 8. (a) Picking up a URL from a TV screen and dropping it into a computer, (b) browsing TV program information on the computer, and (c) operating a VCR by picking up the TV program information from the computer and dropping it in the VCR deck

\section{Implementation Details}

\subsection{Hardware Architecture}

A prototypical InfoStick is depicted in Figure 9. We made it as compact as possible to allow the user to operate it with only one hand. The InfoStick consists of the following items: a SONY CCD-MC1 as a CCD camera to sense video images from a physical environment, three input buttons to "get" / "put" / "select", and a SUNLIKE $16 \times 2 \mathrm{LCD}$ as a display to show the particular information that the InfoStick can "get" / "put" / "select". A PARALLAX BASIC STAMP II is included in the InfoStick as a small computer for controlling the I/O signal and LCD and for exchanging data between the InfoStick and a Mitsubishi AMiTY-CN with RS-232 serial communication protocols. Parallax BASIC Stamps are small computers that run Parallax BASIC (PBASIC) programs. They have programmable $\mathrm{I} / \mathrm{O}$ pins that can be used to directly interface to TTL-level devices, such as buttons, LCDs, speakers, and shift registers. And with extra components, these I/O pins can be connected to non-TTL devices, such as RS-232 networks. The Mitsubishi AMiTY-CN is located on the user's waist. It is a mobile computer for recognizing the $2 \mathrm{D}$ matrix code from the $\mathrm{CCD}$ camera's video image, by using an IBM Smart Capture Card II, and for communicating with other computers on the network. For electric appliances that are not on the network, the nearest IBM PC communicates with other PCs and controls the electric appliances 
through them. SONY VboxII-CI1100s connected to the IBM PCs control electric appliances.

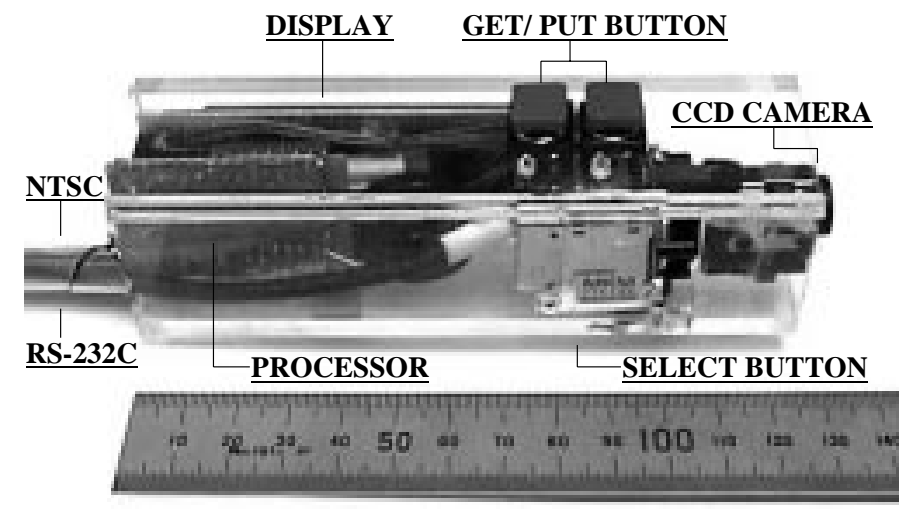

Fig. 9. Configuration of the InfoStick

\subsection{Software Architecture}

Figure 10 illustrates the structure of the software in the InfoStick system. A video image from the CCD camera is first sent to the Marker Reader. The Marker Reader then extracts the black-and-white pattern of the image that the InfoStick is pointing at and analyzes it. If a 2D Matrix Code is found from the image, it is converted to an ID number that will be sent to the InfoStick Controller. Then the controller receives the ID number, it searches for the target object's name and the corresponding available information that the InfoStick can "get" / "put" from the InfoStick DataBase. Marker Reader and InfoStick Controller are implemented in the InfoStick, and the other software modules are in computers connected to the network. The InfoStick DataBase contains the information received by the InfoStick and the ID Table corresponding to the ID number given by the Information Manager on the network. If the user wants to operate one of objects, he can "select" and "get" / "put" the necessary information with the input buttons. In the case of "get", the target information is added into the InfoStick DataBase. On the other hand, in the case of "put", the target physical object is operated by the Machine Controller with the necessary information obtained from the Information Manager.

All code without PBASIC is written in Java and executed on an IBM PC running Windows95. The video capturing class is of special note as it uses 
JDK1.1 Java-native-interface (JNI). The Information Manager and ID table are also Java applications and communicate with other experimental applications through TCP/IP connections. All physical objects used in our applications are directly connected to the Ethernet. The InfoStick, however, uses a wireless local area network. A user therefore can take the InfoStick anywhere within wireless coverage.

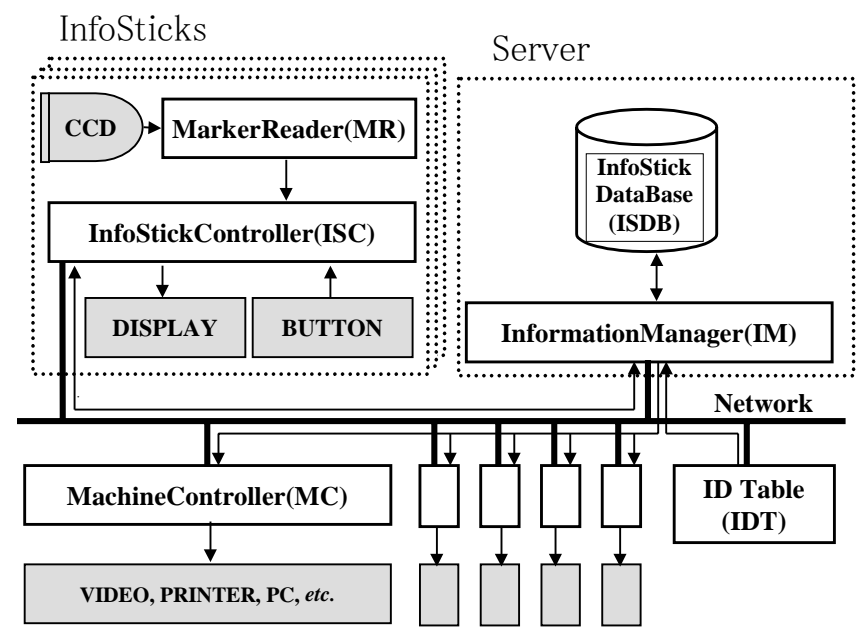

Fig. 10. Software architecture of the InfoStick system

\section{Related Work}

There are several hand-held devices that are designed for operating other digital and physical objects. Most of them, however, aim to operate a single target (e.g., a remote controller for a TV set), rather than to operate several target objects.

The PDA-ITV [4] uses a PDA as a commander for interactive TV. Although it uses two different displays for one task, the roles of PDA and TV are static; PDA acts as a commander only for the TV. Neither seamless manipulation is possible, nor exchanging information between the PDA and ITV is interactive. For example, it is not possible to "pick up" information from the TV screen, and then "drop" it into the PDA. The PaperLink [1] is a computer augmented pen with a video camera that is capable of recognizing text on a printed document. Although PaperLink can pick up information from paper and put it on other paper, it does not support inter object operations. For example, the user can not operate a computer object and paper information with the same PaperLink pen. 
MediaBlocks [5] is a small tag used as a physical container of digital information. The user can virtually attach and carry digital data by using this tag. This system assumes every information appliance has a tag reader/writer, making it different to scale this environment. A user is unable to see the carried data until the tag is actually inserted into a tag reader/writers. Finally, the Pickand-Drop system [2] is a direct manipulation technique that can be used to transfer data between different computers as well as on the same computer. Pick-and-Drop allows the user "pick up" an object from a display and drop it onto another display as if he were operating a physical object. Our InfoStick system is an extension of this system. Although our system also uses the Pickand-Drop metaphor for "get" and "put" operations, the purpose of the InfoStick is to operate not only computers but all physical objects.

\section{Conclusion and Future Work}

We have developed the InfoStick, a new hand-held interaction device that aims to provide a uniform way of operating everyday digital/physical objects. Currently, the InfoStick recognizes the target object by using a combination of attached visual markers and a video camera. When it identifies the ID of the object, the InfoStick searches for the object's name and available information related to that name from the InfoStick DataBase on the network. The idea of deploying visual markers for object-level identification in the environment is aging. However, the use of these markers has advantages and disadvantages. This technique enables to identify an object even if it is not connected to the network, such as a printed material like a poster, a book, or a newspaper, and that visual marker can operate it with easy technology at low cost. On the other hand, a user must always know where the visual marker is. And the camera in the InfoStick needs to be pointed at it.

There are other ways of recognizing objects. They include wireless tags and infrared (IR) beacons. In the case of using a wireless tag, when a receiver approaches within about one meter of the tag, the receiver can recognize its ID number. On the other hand, the IR beacon transmits the ID number to the environment periodically. This beacon covers room-size area and is relatively robust regarding orientation of the sensors. For each method for recognizing objects, there is a different advantage and by using it appropriately, the InfoStick will become more widely used.

In addition, we are planning to provide a "docking station" of the InfoStick for easy information exchange with PCs. When a user "docks" the InfoStick, information stored in the InfoStick is transferred to the PC and the user can exchange them on the PC window with the mouse. The mouse's advantages include being able to "grab", "drag" and "drop" one object from many objects on the same window. On the contrary, when the InfoStick receives a lot of information, it is difficult to select items on the LCD display. If the InfoStick icon pops up on a window when it is put into a docking station connected to a computer, we can release, delete and select objects on the window by using the mouse. (Figure 11). 


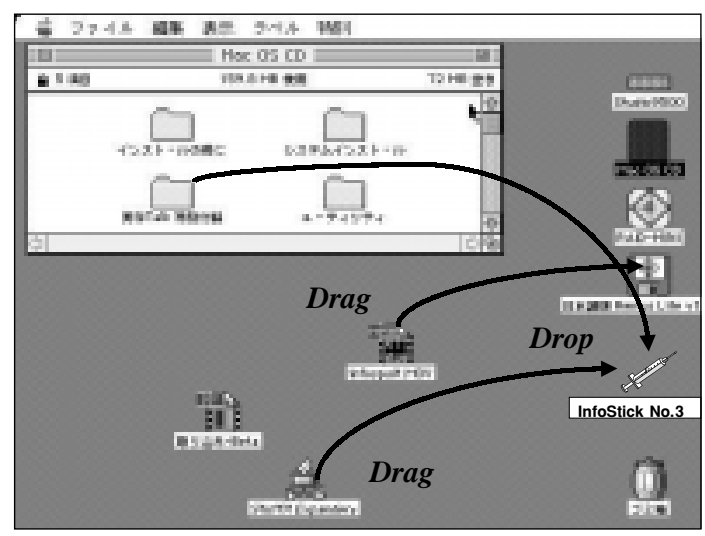

Fig. 11. Drag-and-drop to the InfoStick icon on the computer window

\section{Acknowledgements}

We wish to thank Jun Yamamoto, Nobuyuki Matsushita and Masanori Saitoh from Keio University for their contributions to this project. Several helpful discussions with members of the Sony CSL real-world UI group were also very helpful. We would also like to express our appreciation to Mario Tokoro and Toshi Doi for supporting this research.

\section{References}

1. Toshifumi Arai, Dietmar Aust, and Scott Hudson. Paperlink: A technique for hyperlink from real paper to electronic content. InProceedings of CHI'97, pp.327-333, 1997.

2. Jun Rekimoto. Pick-and-Drop: A Direct Manipulation Technique for Multiple Computer Environments. In Proceedings of UIST'97, pp. 31-39, 1997.

3. Jun Rekimoto, and Masanori Saitoh. Augmented Surfaces: A Spatially Continuous Work Space for Hybrid Computing Environments. In Proceedings of CHI'99, pp. 378-385, 1999.

4. Stott Robertson, Cathleen Wharton, Cathsrine Achworth, and Marita Franzke. Dual device user interface design: PDAs and interactive television. In Proceedings of $C H I^{\prime} 96$, pp. 79-86, 1996.

5. Brygg Ullmer, Hiroshi Ishii, and Dylan Glas. mediaBlocks: Physical Containers, Transports, and Control for Online Media, In Proceedings of SIGGRAPH'98, pp. 379-386, 1998.

6. Mark Weiser. The computer for the twenty-first-century. Scientific American, pp.94104, 1991. 